\title{
Keyword Index for Volume 108
}

\author{
${ }^{212} \mathrm{~Pb}$-TCMC-trastuzumab 2013 \\ $3^{\prime}$ UTR luciferase assay 2070 \\ 4EBP1 2021 \\ 5-aza-2'-deoxycytidine 579 \\ 5-fluorouracil 2505 \\ ${ }^{90}$ Yttrium-PRRT 1440 \\ 9p21.3 1378
}

ABCG2/BCRP 2005

ABVD 49

ACCENT database 784

acetohexamide 570

acute lymphoblastic leukaemia 2334

adenoma 512, 2537

adherence 1515

adhesion 564

adjuvant therapy 1245, 1348, 1978

adolescents 2329

adrenocortical carcinoma 826, 1994

advanced gastric cancer 771

advanced oesophageal cancer 771

advanced ovarian cancer 2264

advanced solid tumours 1998

aerobic glycolysis 1624

aggressive prostate cancer 461

aging 2281

AIB1 1470

AKT 409, 1470, 2021

AKT signalling 2590

alcohol 2407

allergy 2334

allogeneic bone marrow transplantation 1092

alternative splicing 2291

AMD11070 1634

AMG 386503

AMPK 327

anaemia 387

anal cancer 527

anastrazole 2259

androgen deprivation 9

androgen receptor 139,1668

angiogenesis 1126, 2123, 2485

angiogenesis inhibitors 1034

angiopoietin inhibitor 503

animal model 2312

animal studies 64

anoikis resistance 121

ANPEP 420

anti-angiogenic therapy 250, 319

antibody 1449

antileukaemic 1870

anti-vascular 1306

ANXA11 rs1049550 1862

anxiety 1012

apoptosis 170, 395, 1460, 1648, 2070, 2097

ARID1B 2056

aromatase inhibitor 1408, 1515, 2259

array Comparative Genomic Hybridisation 2172

arthroplasty 1883

Asian Americans 1508

aspirin 1178, 1189

astrocytoma 587

atelocollagen 836

ATFs 881

attitudes 831,1602

autoantibodies 107, 2045 awareness 292

axitinib 1571

Axl 621

bafilomycin 2312

Bayesian 762

$\beta$-blockers 2485

$\beta$-catenin 2130

Bcl-2 phosphorylation 1870

Bcl-xL 653, 2312

BEACOPP 49

benign breast disease 199

benign prostatic hyperplasia 1778

benign tumours 193

bevacizumab 493, 1052, 1316 1566,2549

BH3 mimetics 1870

biliary obstruction 1846

Bim 442

biomarkers 99, 107, 139, 342, 361, 395, 420, 644, 662, 908, 924, 932, 1052, $1100,1298,1316,1712,1822,1846$, $1854,1925,2448$

bisphosphonates 163

bladder 1534

bladder cancer 973, 1470, 1854 2372

body mass index 1891, 1408, 2390

bone markers 2565

bone metastasis $163,2525,2565$

bortezomib 762

BRAF 1634, 1757, 2153

BRAF inhibitor 924

BRAF mutation 1495

brain-derived neurotrophic factor 12

brain tumour 2367

brain tumour epidemiology 715

brain tumour stem cells 2005

BRCA1 461, 1695, 1732, 2601

BRCA1 and BRCA2 genes 1579

BRCA1 immunohistochemistry 1231

BRCA1 mutation 2172

BRCA1/2 testing 1012

BRCA2 1732

BRCAness 2172

breast cancer $163,188,285,549,557$, $602,820,848,866,873,1042,1195$, $1408,1480,1515,1579,1602,1807$, $1985,2033,2045,2172,2241,2250$ 2291, 2304, 2399, 2426, 2442, 2525.

breast cancer diagnosis 351

breast cancer radiotherapy 179

breast-conservative therapy 820

breast-conserving surgery 812

breath analysis 941

Breslow 1641

BTG2 973

bystander effect 91

CA125 antigen 2448

CA 19-9 1684

c-Abl 1143

cachexia 486

CA IX 402, 613

cancer at a young age 1525

cancer heritability 2419

cancer inflammation 2116

cancer management 2442 cancer pain 259

cancer registration 691, 699

cancer stem cells 1449, 2525

cancer survivors 831, 1525, 2407

capecitabine 469, 2505

carbonic anhydrase IX 429

carcinoembryonic antigen 662

carcinoid 301

carcinoid tumour 1838

carcinoma 638, 1553, 2537

carcinoma of unknown

primary 39

cardiovascular risk 9

case-control 222, 240, 1899

CCID 564

CCL15 99

CD24 1449

CDA 2505

CDK6 1143

CDKN2A 1378, 2542

cediranib 493, 1316

celecoxib 2272

cell cycle 2021, 2495

cell cycle arrest 1648

cell lines 1801

cell migration 973

cell penetrating peptide 2495

cell proliferation 836

cervical cancer 1348, 1957, 2116, 246

cervical intraepithelial neoplasia 234

cervix 908

cetuximab 771

checkpoint blockade 1560

CHEK2 461, 2601

chemokine 1634

chemoresistance 1079

chemotherapy $39,265,395,486,1027$,

$1071,1425,1684,1771,1931,2005$

2063, 2264, 2250, 2485

childhood brain tumour 2346

childhood cancer 1280, 1907

childhood CNS tumours 1907

childhood leukaemia 1899, 2339

children 2334, 2346

CHIPS 587

CHK2 2601

CHOP 1340

chorionic gonadotropin 1810

circulating microRNA 932

circulating tumour cells 549, 791, 1358

2426

cisplatin 265, 579, 859, 1245, 1695, 1994

cixutumumab 826

CK-19 549

class III $\beta$-tubulin 82

clear cell 1553

cleaved caspase-3 2097

cleaved PARP 2097

clinical benefit response 1021

clinical outcome 1042

clinical response 285

clinical trials 748, 866

clinicopathologic 1508

cluster analysis 2354

c-Met 2142

c-Myc 653

CNS development 2130

CNS PNET 2130

COBAS 2164 coffee 2381

cohort study 708, 1168, 1178, 1879, 1883,2390

collagen genes 964

collagen receptor 163

colon 107

colon cancer 784, 791

colonic neoplasms 1425

colorectal cancer 278, 493, 662, 668, 681, $1149,1316,1368,1432,1502,1508$,

$1566,1712,1720,1750,1757,1862$

1891, 1978, 2088, 2097, 2106, 2153 .

$2537,2542,2549$

colorectal neoplasm 213, 775

colospheres 1720

combination 1393

combination immunotherapy 1998

common alleles 2610

common genetic variants 1378

communication 1402

communication inequalities 1949

competing risk 1534

complement 5a 1846

comprehensive geriatric assessment 1936

consultations 686

conventional adenocarcinoma 613

COP1 2495

cost 1225,2250

cost-effectiveness $9,1579,2250$

COX-2 91, 1106, 2272

COX-2 inhibitor 319

Cox 784

C-reactive protein 1891

cross-sectional studies 292

CRPC 1668

cryptic 1113

CTC 2426

curriculum 1936

CXCR4 1634

CYFRA 21-1 1684

cytarabine 983

cytokeratin 1704

cytokines 1306, 1801

cytotoxic T cells 1092

cytotoxic T- lymphocyte antigen 4

(CTLA-4) 1560

D2 gastrectomy 1245

database 721

DCIS 812,1593

decision impact 2250

decision support techniques 1810, 2448

delay 686,1280

delivery of health care 14

dendritic cells 1092

DEPArray 1358

dephosphorylation 2590

depression 1012

desmoplasia 1

detection marker 1119

DFMO 512

diabetes 222, 1778, 727

diabetes mellitus 1182

diagnosis 25, 686, 941, 1149, 1332, 2329

diagnostic 1712, 1925

diagnostic assay 1157

diagnostic interval 1280

diagnostic marker 163, 2299

diarrhoea 1027 
dichloroacetate 72, 1624

dielectrophoretic cell sorting 1358

diet 188, 205, 512, 831

diffuse-type gastric cancer 932

digital pathology 602

discrete choice experiment 533

disease-free survival 2573

disease progression 2565

Dkk2 2070

Dkk-3 1659

DNA adducts 334

DNA content analysis 873

DNA damage signalling 748

DNA methylation 131, 370, 420, 2033

DNA mismatch repair 1495, 2079

DNA repair 748

docetaxel 265, 771, 1079, 1994

docosahexaenoic acid 486

Down syndrome 2334

doxifluridine 1245

DPYS 2505

drug resistance 1810

ductal intraepithelial neoplasia 1593

dying 1942

E2F1 1470

E3 ligases 2495

E7 450

early deaths 681

early diagnosis 2045

early stage 1348

EBP1 557

E-cadherin 402

eEF1A2 2304

effect size 1790

eflornithine 512

EGFR 115, 469, 1743

EGFR family 2291

EGFR signalling 1765

EGFR-targeted therapy 668

eicosapentaenoic acid 486

elderly $311,1534,1936$

elderly patients 519

embolisation 1252

end of life care 1273

endometrial cancer 183, 1021, 1267, 1771

endoplasmic reticulum stress (ER stress) 1340

England 775

EpCAM 881, 1480

ependymoma 2367

EPIC 708

epidemiology 179, 183, 699, 721, 1168,

$1189,1899,1907,2334,2346,2367$

epidural dexamethasone 259

epidural methadone 259

epigenetic alteration 2557

epitope 1113

epothilones 82

Epstein-Barr virus 2557

Erbb4 2291

ERCC-1 115, 1238, 1695

ERK5 149

erlotinib 469

erythrocyte sedimentation rate 387

esophageal cancer 1822

estradiol 199

estrogens 199

estrone 199

EUS-FNA 1488

evaluation 1942

everolimus 1616, 1771, 2478

evidence 1225

EWOC 762

exemestane 2259 extracellular matrix 964

extranodal NK/T-cell lymphoma 380

ex vivo model 1720

FACS 2070

family history 1502

fasting blood glucose 380

fatigue 49

female 1534

fertility 1602

FISH 2582

flavonoids 1168

FLO 519

FLOT 519

fluid intake 2372

fluorescence in situ hybridisation 668

FOLFIRI 1566

FOLFOXIRI 2549

follow-up marker 1119

follow-up studies 2455

FoxO3a 442

FOXP3 155

FPR 1587

France 775

\section{GADD34 1340}

Gas-6 621

gastric adenocarcinoma 653

gastric cancer 121, 395, 941, 1245, 1324,

1495, 2557

gastric phenotype 613

gastroesophageal cancer 519

gemcitabine 469, 766, 1393, 2063

general practitioner 686

genetic polymorphism 1695, 2372

Genistein 2070

genitourinary toxicity 1964

genomic alteration 1732

genotype 676

geriatric oncology 1936

gestational trophoblastic neoplasia 1810

$\gamma \mathrm{H} 2 \mathrm{AX} 2021$

glioblastoma 2005, 2142

glioma $64,715,1189,2178,2516$

GLUT-1 402

glutathione S-transferase 676

glycolysis 72,170

glycosylation 1113

GNAS 951

graft vs tumour reaction 1092

growth factors 1801

GWAS 2419

HAART and smoking 1173

haematological malignancy 2390

hazard ratio 2299

HCC 99

HDAC1 1648

HDAC 342

head and neck cancer 1178

head and neck carcinogenesis 1157

health communication 1949

health disparities 1949

health information avoidance 1949

Health-related quality of life (HRQoL)

$$
49,1784
$$

height 2390

hENT1 1488

hepatitis B reactivation 1931

hepatitis B screening 1931

hepatoblastoma 334

hepatocellular carcinoma $21,72,222$,

$442,1182,1252,1830$

HER2 668, 873, 1807, 1985
HER4 2291

hereditary 1732

hereditary cancer 1579

herpes zoster 721

HES 1273

heterogeneity 479

H-HPV 613

HIF 1133

Hif- $1 \alpha 429$

high-voltage overhead power lines 1899

HIPEC 1432

hip prosthesis 1883

histone deacetylase inhibitors 748

histopathology 866

HNSCC 1648

Hodgkin lymphoma 49, 1163, 2399

hospital admissions 1273

HPV 908, 1332

HPV16 229, 240, 450

HPV16 + cervical cancer 859

HPV16 + E6 oncogene 859

HPV 16 + HNSCC 859

HPV infection 1157

HPV integration 229

H.pylori gastritis 1113

hTERT 278, 2272

human fecal microbiome 1163

human-in-mice model 2525

human papillomavirus 240

hyaluronic acid 1

hyaluronidase 1

hygiene hypothesis 1163

hypermethylation 1368

hypoxia 1133

ibuprofen 1178

ICAM-1 564

IDH1-R132H 2142

IGF-1 199

IGF-1R pathway 826

image analysis 602

imaging 662

immune microenvironment 914

immune/inflammatory cell

infiltration 914

immunisation 1092

immunohistochemistry $602,924,1267$, $1830,2164,2582$

immunotherapy 1288, 1560, 2281

incidence $1899,2367,2381$

individually tailored breast cancer screening 2241

infections 2478

infectious disease 2346

inflammation 997

information provision 1602

information technology 2433

ING1 1143

inguinal lymph node 527

inherited susceptibility 2178

integration 629

integrin 1460,2516

interaction 1173,1778

intercellular adhesion molecule 2

(ICAM2) 1817

interleukin-1 $\beta 2063$

interleukin-6 1891, 2063

interstitial fluid pressure 1

intracranial xenografts 64

intraperitoneal chemotherapy 1231

intra-tumour 479

intrauterine devices 234

intrinsic subtypes 1480

intronic deletion 438

invasion 99, 2516

invasion assay 2070

invasive breast cancer 542

ipsilateral recurrence 812

iron chelators 409

isoxsuprine 570

ITIH3 1846

Japanese survey 629

Kaplan-Meier 784

Kaposi's sarcoma risk 1173

Ki-67 271, 1593

Ki-67 antigen 1838

kinase inhibitor 334

KRAS 115, 951, 1757, 2153

KRAS mutation 1495

late effects 1525

lead time 1971

lenalidomide 1801

letrozole 1587, 2259

leukaemia 2390

leukocytes 1306

life expectancy 1971

lifestyle factors 1508

LIFR rs3729740 1862

liquid biopsy 2426

Listeria monocytogenes 2281

liver 676

liver cirrhosis 21

liver function 21

liver metastases 1566, 2549

Liverpool Care Pathway 1942

liver toxicity 2549

LKB1 327

lncRNA 2419

lobular carcinoma 285

lobular endocervical glandular hyperplasia 613

localised prostate cancer 271, 2464, 2582

log-normal 784

longitudinal 2407

long-term effects 179

long-term medical consequences 1525

low-dose tamoxifen 1593

low-grade mucinous appendiceal neoplasm 951

LRIG1 1765

lung 91

lung cancer $629,1288,1879$

lymphatic endothelium 564

lymphendothelial intravasation 570

lymph node 2106

lymph-node metastasis 1267

lymphoma 2390

Lynch syndrome 2079

macrometastasis 1267

macrophage inhibitory cytokine-1 1079

maintenance therapy 2264

male breast cancer 959, 2259

male infertility 1004

malignant mesothelioma 621, 1743

malignant neoplasm 193

malignant outcome 1324

malignant peripheral nerve sheath tumour 964

malignant potential 2033

mammalian target of rapamycin

inhibitor 1021

mammographic calcifications 812

MAPK 1460

margins 285 
matrix metalloproteinase 1149 MCF7 cells 2304

medical record linkage 1883

melanoma 924, 1560, 1634, 1641, 1998, 2164

melatonin 442, 2005

men 533

Merkel cell polyomavirus 629

mesothelioma 1340

MET 1100

meta-analysis 39, 64, 798, 2299, 2478

metabolic syndrome 222

metabolism 327

metalloproteinases 964

metastasectomy 1425

metastasis $265,493,549,1079,1432$

$1541,1634,1668,1704,2106,2470$ metastatic breast cancer 1052, 2281

metastatic colorectal carcinoma 503

metastatic solid tumours 1061

methotrexate 1810

methylation 1750, 2542

MET inhibitor 21

metronomic chemotherapy 250

mFOLFOX6 493

mFOLFOX 791

MHC-II 2097

microarray 107, 370

micrometastasis 1267, 2426

microRNA 131, 361, 1817, 1822, 1925

microRNA-18a 653

microRNA profile 1712

microsatellite instability 1750,1757 , 2079

microtubules 82

midkine 1854

migration 99, 587

minimal deviation 613

mir-124-3 131

miR-1246 644

miR-125b 1817

miR-1260b 2070

mir-143 149

miR-145 2573

mir-203 1143

miR-205 1668

miR-29c 964

miR-663 2304

miR-744 2304

miR-874 1648

miRNA 1133, 1668

miRNA-183 1659

mismatch repair 983

mitochondria 2485

mitochondrial peptides 587

mitomycin-C 1245

mitotic count 1838

mitotic spindle checkpoint 2013

MLH1 983

MMR status 1238

molecular 1553

molecular characterisation 1157, 2426

molecular tumour markers 2573

morbidity 32

mortality $179,188,1515,1534,1757$

mortality trends 2354

MSH2 983

MTHFR 2505

mTOR 429, 1616, 2021

mTOR inhibition 1771, 2478

mTOR pathway 826

MTS assay 2070

MUC1 1113

MUC1 aberrant glycoforms 2045

mucinous adenocarcinoma 1978 mucinous histology 1978

multicentre study 1495

multidisciplinary case conference 2433

multidisciplinary teams 2442, 2433

multiparametric flow cytometry 873

multiple myeloma 170, 1624, 1801

multi-state Markov model 542

multi-state multi-variable model 2241

mutation 629, 1368

mutation analysis 1743

Myeloid-derived suppressor cells 228

myofibroblasts 2106

NBN 461, 2601

NBS1 461, 2601

NDRG1 409

needle biopsy 271

negative regulators 597

neoadjuvant chemotherapy 285,519 ,

$866,1566,1587,1807,1957,2172$,

2464

neoplasm 14, 292, 1810, 1883, 2381, 2537

neratinib 1985

nestin 2142

neuroblastoma 2485

neuroendocrine 301

neuroendocrine tumour 1298, 1440,

1838

neurofibromatosis 193

neurofibromatosis type 1964

neutrophil/lymphocyte ratio 1677

next-generation sequencing 479

NGAL 2537

NGR-hTNF 58

nifedipin 570

nitrate 205

nitrite 205

non-aspirin NSAIDs 1189

non-small-cell lung carcinoma 1415

non-targeted response 1106

Norway 1525

Notch3 1488

NSCLC 265, 1695

obesity 222, 727, 1408

oesophageal squamous cell carcinoma 644

oesophago-gastric cancer 25

oestrogen receptor 155

oestrogen sulphotransferase 1415

off-target effects 450

older women 1042

oncologists 1602

oncologists' views 2264

oncology 1071, 1273

oncolytic 245

one-carbon metabolism 183

oral contraceptives 234

oral squamous cell carcinoma (OSCC)

370,1817

oral tongue cancer 638

oropharyngeal 1332

osteosarcoma (OS) 836

osteotropism 2525

outcome 887

ovarian cancer $250,881,1231,1553$,

1732, 2039, 2448

ovarian cancer relapse 32

ovarian neoplasm 2448

overall survival 32,2565

overdiagnosis 1971

oxaliplatin 115, 265, 676, 1238, 1978

oxidative phosphorylation 72

oxidative stress 848 p16 immunohistochemistry 1157

p16 ${ }^{\text {INK4A }} 229$

p28 1061

p53 107, 1061, 2495, 2573

p53 mutant 873

paclitaxel 1985, 2013

PAK1 557

palliative care 1273

pancreatic cancer $361,469,766,914,997$, 1684, 1846, 2056, 2063

pancreatic ductal adenocarcinoma 1

pancreatic neoplasms 1168

paraganglioma 429

parent-of-origin effects 2610

parity 234

paternal occupation 1907

pathological complete response 285 , 1587

pathologic response 2549

patient care planning 14

patient reported outcomes 301, 1571

patients' views 2264

PDK1 170

penetrance 2610

penile cancer 229

peptide vaccine 1260

perceived probability of genetic predisposition to cancer 1012

period analysis 699

periodate 1113

peritoneal carcinomatosis 1432

persistent human papillomavirus

infection 234

personalised cancer treatment 1358

PET 342

PF-3512676 1998

Phaeochromocytoma 429

pharmacodynamic 58, 1298

pharmacokinetics 1393

pharmacology 342,1071

phase I trial 762, 826, 1260

phase II 1994

phase III trial 1957

phosphorylation 139, 2590

physical activity 798, 831, 2407

PIAS3 653

PIGR 1846

PIK3CA 1807

pineoblastoma 2130

plasma 361,1822

plasma marker 163

platinum 2039

podocalyxin-like protein 2321

polyamines 512

polymorphism 438, 1126

polyunsaturated fatty acids 486

pooled analysis 727

population-based 1195

population-based data 691

population-based study 775

population studies 2455

positive predictive values 25

post-bereavement survey 1942

postoperative relapse 791

potential drug-drug interactions 1071

PP2A subunits 2590

predictive 1052, 1925

predictive biomarker 1238

pregnenolone sulphate 1415

premalignant 2033

primary care $25,1280,2329$

proadifen 570

progesterone receptor 1415

prognosis $131,155,370,380,395,420$, $621,901,914,1100,1119,1133,1332$,

$1593,1810,1830,1838,1925,2153$,

2321,2542

prognostic biomarker 387, 1677

prognostic factors $271,681,1324,1480$,

2088, 2312, 2470, 2582

prognostic marker 278, 2299

programmed death-1 (PD-1) 1560

proliferation 1470

promoter methylation 2005

pro-opiomelanocortin 1704

prophylactic antiviral therapy 193

prospective study 213,2381

prostate cancer $9,139,149,409,420,597$

708, 1079, 1225, 1659, 1668, 1778,

1784, 1879, 1925, 1964, 1971, 2123,

$2381,2565,2573,2590,2601$

prostate cancer screening 533

prostatectomy 1784

prostatic neoplasm 2354

prostatitis 1778

protein phosphatase type 2A 2590

proteomics 351

proton beam 1225

proxy 1942

PSA 533, 2601

psychology 1004

PTEN 409, 438, 2582

publication bias 64

pyrosequencing 2164

Quadruple index 2153

quality of care 1942

quality of life $301,1225,1571,1790$

quaternary cytoreduction 32

radiation 91, 1106, 1225

radiation-induced neoplasm 1579

radiation-related heart disease 179

radiation-related lung cancer 179

radiation therapy 1964

radical hysterectomy 1348

radical surgery 1957

radioimmunotherapy 859

radioresistance 1817

radiotherapy $812,1593,1784$

RAF inhibitors 1298

Raf kinase inhibitor protein 2088

randomised 1402

randomised trial 812

rates 2455

RCC 2070

real-time RT-PCR 2070

RECIST 1252

record linkage 193

rectum 107

recurrence 188, 1119, 1515

referral 686,1149

REG 395

registration errors 691

regression 1790

regulatory RNA 2419

relative survival 691

renal cancer 205, 798, 1133, 1616

renal cell carcinoma 131, 205, 311, 319,

$327,387,887,901,1034,1126,1260$

$1541,1571,2470,2478$

resection 1425

resistance 2039

response 1440

resveratrol 2304 
2646

risk of cancer 193

risk stratification 2573

RKIP 2088

RNAi 245

\section{S1 1870}

sagopilone 82

Sanger 2164

sarcomas 2455

sarcopenia 1034

SCC 1332

screening 245, 1579, 1971, 1508, 2045, 2601

second tumours 2455

secretome 1854

segregation analysis 2610

semen cryopreservation 1004

sensitivity 542

sensitivity and specificity 2299

sensor 941

sentinel lymph node 527, 1267

serum 1149, 1712

serum microRNA 644

sex chromosome aneuploidy 959

sexual behaviour 234

sexual function 49

FRP1 2070

side effects 1784

signal transduction 1298

simulation 691

simulation model 1579

single-cell analysis 1358

single-nucleotide polymorphisms 887

sinusoidal obstruction syndrome 676

siRNA 450

keletal-related events 2565

sleep 213

SMAD2 409

SMAD4 1659

Smad4 2070

smoking 708, 2407

SNPs 2601

social contact 2346

social security benefits 1525

socioeconomic status 1907, 2339

soft-tissue sarcoma 1677

sojourn time 542

solid tumours 1393, 1931

soluble tumour necrosis factor receptor 21891

somatostatin receptor 12557

sorafenib $72,311,334,1571$

Sox11 2142

spheroid 579

spinophilin 1830 splice-switching oligonucleotide (SSO) 2291

SPRED1 597

SPRED2 597

squamous cancer 527

squamous cell carcinoma 638

Src 1143

SRC3 2039

stable disease 1021

stage III colon cancer 1238

staging 527, 1195

STAT3 653

statins 715

stem cell 1765

stem-like cell 2516

stereology 2116

steroid sulphatase 1415

stress reactions 1012

stromal resistance 1

study population 699

sulindac 512

sunitinib 319, 762, 887, 1034, 1126, 1393 ,

1541,2470

supportive care 2264

supradiaphragmatic radiotherapy 2399

surgical-pathological risk factor 1348

surgical treatment 1119,1566

survival $387,461,681,775,784,1195$,

$1440,1502,1508,1541,1641,1757$,

$2164,2339,2367,2470$

survival analysis 699

survival rate 292

survivin 653,848

survivors 14

survivorship 1163

Sweden 1541

SWI SNF 2056

Sylamer 450

symptom control 1027

symptoms 25

synthetic lethality 983

systematic review 39, 2299

systems pathology 602

TACE 1252

TAE 1252

tamoxifen 557, 1408, 1515

targeted chemotherapy 1862

targeted immune modulator 1998

targeted therapy $311,924,1079,1553$, 1616,1743

taxotere 1994

teachable moment 2407

teenagers 2329

telemedicine 2433 telomerase 1368, 2272

temozolomide 64

temsirolimus 2478

testosterone 199

TGF- $\beta 1106$

TGFBR 1106

therapeutics 479

therapy 997, 1553

therapy response 2088

three-dimension 1720

timing radiotherapy 820

tissue biomarkers 351

tissue microarray (TMA) 420, 1340

tivantinib 21

TLR9 1616

Toll-like receptor 5638

TOPflash 2070

toxicity 1560,1784

training 1936

transcriptional regulation 1143

transforming growth factor- $\beta 91$

translational research 2241

trastuzumab 1807

treatment outcomes 1034

trebananib 503

Treg 155

tremelimumab 1998

trial participation 1402

trichostatin A 579

triple-negative breast cancer 1100, 2172

tropomyosin-related kinase B 12

TUBB3 82

tumorigenicity 579

tumour 342

tumour-associated macrophages 1288 ,

2116

tumour-associated neutrophils 2116

tumour-associated stroma 2097

tumour board 2433

tumour cell invasion 755

tumour dissemination 32

tumour grading 1838

tumour growth 334

tumour hypoxia 402

tumour immunity breast cancer 155

tumour immunology 1288

tumour invasiveness 402

tumour microenvironment 755, 1677

tumour spheroid 570

tumour spheroid intravasation 564

tumour suppressor 973,1765

tumour suppressor gene 1368, 2056, 2557

twin study 1163

TYMS 2505 tyrosine kinase inhibitors 250, 311, 1541, 1985

TYRP1 1641

ubiquitination 2495

UDP-glucuronosyltransferase 2372

ulceration 1641

ultrasonography 2448

underground mining 1879

unresectable pancreatic cancer 1488

uPA inhibitor 766

upper urinary tract 2312

urine 1854

urothelial 1534

urothelial carcinoma 1368, 2312, 2321

uterine cervix cancers 402

uterine sarcoma 727

V600E 924

vaccine therapy 1260

validation study 901

Van-Gogh-like 21750

vascular disruptive agents 250

vascular endothelial growth factor 1052

vascular endothelial growth factor receptor 1260

vascular targeting agent 58

vasohibin-1 2123

VEGF 429, 1052, 1126, 1316, 1460

VEGF-A 1034

VEGF inhibitors 250

VHL 1133

vincristine 2485

viral hepatitis 1182

viral load 229

virotherapy 245

virtual teams 2433

volatile organic compound 94

weight loss 831

western blot 2070

WNT 1750

WNT pathway 2130

women 213

worldwide 240

xanthenones 1306

xenografts $334,848,1306,1704$

Y-box binding protein-1 (YB-1) 836

younger adult 1602

YWHAZ (14-3-3६) 1324

zinc-fingers 88 